\title{
A risk score to predict the incidence of prolonged air leak after video-assisted thoracoscopic lobectomy: An analysis from the European Society of Thoracic Surgeons database

\author{
Cecilia Pompili, MD, ${ }^{\mathrm{a}}$ Pierre Emmanuel Falcoz, MD, ${ }^{\mathrm{b}}$ Michele Salati, MD, ${ }^{\mathrm{c}}$ Zalan Szanto, MD, ${ }^{\mathrm{d}}$ and \\ Alessandro Brunelli, MD
}

\begin{abstract}
Objective: The study objective was to develop an aggregate risk score for predicting the occurrence of prolonged air leak after video-assisted thoracoscopic lobectomy from patients registered in the European Society of Thoracic Surgeons database.

Methods: A total of 5069 patients who underwent video-assisted thoracoscopic lobectomy (July 2007 to August 2015) were analyzed. Exclusion criteria included sublobar resections or pneumonectomies, lung resection associated with chest wall or diaphragm resections, sleeve resections, and need for postoperative assisted mechanical ventilation. Prolonged air leak was defined as an air leak more than 5 days. Several baseline and surgical variables were tested for a possible association with prolonged air leak using univariable and logistic regression analyses, determined by bootstrap resampling. Predictors were proportionally weighed according to their regression estimates (assigning 1 point to the smallest coefficient).
\end{abstract}

Results: Prolonged air leak was observed in 504 patients $(9.9 \%)$. Three variables were found associated with prolonged air leak after logistic regression: male gender $(P<.0001$, score $=1)$, forced expiratory volume in 1 second less than $80 \%(P<.0001$, score $=1)$, and body mass index less than $18.5 \mathrm{~kg} / \mathrm{m}^{2}$ $(P<.0001$, score $=2)$. The aggregate prolonged air leak risk score was calculated for each patient by summing the individual scores assigned to each variable (range, 0-4). Patients were then grouped into 4 classes with an incremental risk of prolonged air leak $(P<.0001)$ : class A (score 0 points, 1493 patients) $6.3 \%$ with prolonged air leak, class B (score 1 point, 2240 patients) $10 \%$ with prolonged air leak, class $\mathrm{C}$ (score 2 points, 1219 patients) $13 \%$ with prolonged air leak, and class D (score $>2$ points, 117 patients) $25 \%$ with prolonged air leak.

Conclusions: An aggregate risk score was created to stratify the incidence of prolonged air leak after video-assisted thoracoscopic lobectomy. The score can be used for patient counseling and to identify those patients who can benefit from additional intraoperative preventative measures. (J Thorac Cardiovasc Surg 2017;153:957-65)

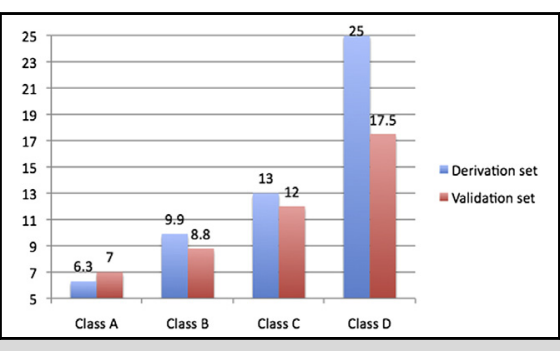

Incidence of PAL after VATS lobectomy according to the class of risk.

\section{Central Message}

The risk score predicting PAL after VATS lobectomy can be used to implement preventative measures and for patient-informed counseling.

\section{Perspective}

An aggregate risk score for PAL specific for VATS lobectomy has been developed. This score can be used to identify high-risk patients who may benefit from intraoperative preventative measures (eg, sealants, buttressing material) aimed at reducing the occurrence of this complication with a potential clinical and financial benefit.

See Editorial Commentary page 966.
Previous articles have tried to identify risk factors for prolonged air leak (PAL) after lung surgery ${ }^{1-3}$; however, there is no risk score for patients undergoing video-assisted thoracoscopic surgery (VATS). Reliable information on the risk of

\footnotetext{
From the ${ }^{\mathrm{a} D e p a r t m e n t}$ of Thoracic Surgery, St James University Hospital, Leeds, United Kingdom; ${ }^{b}$ Department of Thoracic Surgery, University Hospital Strasbourg, Strasbourg, France; ' ${ }^{\text {Unit }}$ of Thoracic Surgery, Ospedali Riuniti, Ancona, Italy; and ${ }^{\mathrm{d}}$ University of Pecs, Pecs, Hungary.

This study represents an investigator-sponsored study, which was supported by a grant from C.R. Bard, Inc. The funder had no role in the data analysis, interpretation of the data or preparation, review, or approval of the manuscript.

Read at the 96th Annual Meeting of The American Association for Thoracic Surgery, Baltimore, Maryland, May 14-18, 2016.
}

these patients developing PAL after lung resection would help to inform the need for intraoperative preventative measures (eg, pleural tenting, surgical sealants) to minimize the occurrence of this complication. Also, this information 


\section{Abbreviations and Acronyms \\ BMI = body mass index \\ DLCO $=$ carbon monoxide lung diffusion capacity \\ ESTS $=$ European Society of Thoracic Surgeons \\ FEV1 = forced expiratory volume in 1 second \\ PAL = prolonged air leak \\ VATS $=$ video-assisted thoracoscopic surgery}

Scanning this QR code will take you to a video for the article. To view the AATS 2016 Webcast, see the URL next to the video thumbnail.

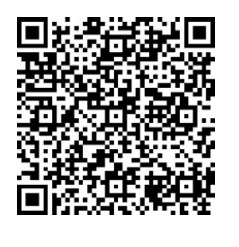

can help set realistic expectations about the duration of chest tubes and hospital stay, which remains a main driver in patient satisfaction after minimally invasive operation.

Furthermore, the use of minimally invasive approaches has expanded the inclusion criteria for lung resection, with an increasing proportion of patients with poor lung function and advanced age undergoing operation. ${ }^{4-8}$ The change in the case mix of surgical candidates traditionally submitted to thoracotomy warrants the development of a risk model.

Although video-assisted thoracoscopic lung resection is associated with a lower incidence of complications compared with thoracotomy, ${ }^{9}$ the frequency of PAL appears similar in the 2 groups of patients, as shown in the 2 recent case-matched analyses from the European Society of Thoracic Surgeons ${ }^{10}$ (ESTS) and the Society of Thoracic Surgeons ${ }^{11}$ general thoracic surgery databases. The objective of this study was to develop an aggregate risk score for predicting the occurrence of PAL after VATS lobectomy from a large cohort of patients registered in the ESTS database (Video 1).

\section{MATERIAL AND METHODS \\ Data Source}

The features and scope of the ESTS database have been described. ${ }^{10,12}$ Briefly, the ESTS database is an online voluntary general thoracic surgery database that is free and publicly available to all ESTS members. The ESTS database is compliant with the International data protection assurance regulation. No Ethical Committee review is required to submit data to the database. Data can be inputted into the database online by individual surgeons or data managers, and institutional dataset can be imported annually through an automated procedure involving variable matching. The database was started in 2007 and currently collects data and information from more than 200 European hospitals. All variables and outcomes in the database have been standardized and defined a priori. Definitions of variables are reported in the joint Society of Thoracic Surgeons ESTS article on standardization of definition of variables in the respective registries. ${ }^{12}$

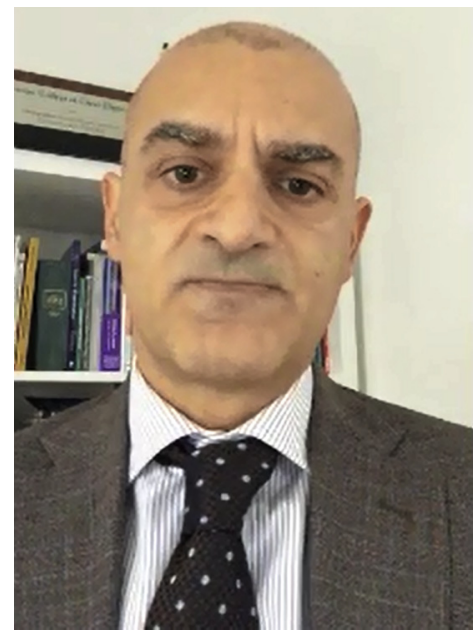

VIDEO 1. Dr Alessandro Brunelli, senior author, and consultant thoracic surgeon in Leeds, United Kingdom, describes the rationale, main finding, and implications of this study. Video available at: http://www. jtcvsonline.org/article/S0022-5223(16)31804-9/addons.

The ESTS database is not systematically audited, and only samples of data from participating centers eligible for the Institutional Accreditation Program are subject to audit. These represent only $10 \%$ of all centers contributing to the database.

\section{Study Cohort}

This is a retrospective analysis performed on 5069 VATS lobectomies (99 bilobectomies and 4970 lobectomies) registered in the ESTS database from July 2007 to August 2015. With the aim to eliminate a learning curve effect, only patients operated on in units contributing more than 20 VATS lobectomies were used for this analysis (these represented $90 \%$ of the total VATS lobectomies in the ESTS database). The number of VATS lobectomies registered from each center varied from 21 to 360 (19 centers contributed $>100$ patients). They represent $13 \%$ of all lobectomies registered in the ESTS database during the same period $(38,672$ lobectomies in total). The ESTS database does not collect information about the modality of VATS approach (ie, number of ports, anterior or posterior approach, fissureless technique) or the occurrence of conversion to thoracotomy. Furthermore, it does not distinguish between video-assisted and robotic-assisted procedures. A more specific portion of the database dedicated to minimally invasive lung resection has been recently implemented to overcome these limitations.

For the purpose of this investigation, patients undergoing anatomic or nonanatomic sublobar resections or pneumonectomies were excluded from the analysis. Likewise, patients undergoing VATS lobectomies and additional extended procedures, such as chest wall or diaphragm resections (31 patients) and sleeve resections (25 patients), or requiring postoperative mechanical ventilation (34 patients) were not included in the analysis, because these characteristics were thought to influence the occurrence and duration of air leak or to alter the pulmonary mechanics. Also, 49 patients died in the hospital or within 30 days from operation (30-day mortality rate $1 \%$ ) and were excluded from the analysis.

\section{Data Collection and Statistical Model}

According to the definition in the database, PAL was defined as an air leak more than 5 days. ${ }^{12}$ PAL represents the main end point of this analysis. The ESTS database does not collect the duration of air leak expressed as continuous numeric variable.

The following baseline and surgical variables were screened for a possible association with PAL: age, sex, forced expiratory volume in 
1 second (FEV1) expressed in percentage of predicted value, body mass index (BMI), Eastern Cooperative Oncology Group score, diabetes, presence of coronary artery disease, neoadjuvant chemotherapy, and side and site of lobectomy.

For the purpose of this analysis, cardiopulmonary complications were considered those occurring during the hospital stay or within 30 days from operation and included the following: respiratory failure requiring mechanical ventilation for more than 24 hours, pneumonia, atelectasis requiring bronchoscopy, pulmonary embolism, pulmonary edema, adult respiratory distress syndrome, acute myocardial infarction, atrial fibrillation requiring medical or electrical cardioversion, cardiac failure, and stroke.

Missing data were imputed by averaging the nonmissing numeric values, or choosing the most frequent value in the categoric variables. All variables used for this analysis had less than $10 \%$ missing data. Carbon monoxide lung diffusion capacity was present in only $30 \%$ of patients and could not be used for constructing the model. The normal distribution of numeric variables was first assessed by the Shapiro-Wilk normality test.

To develop a risk score for PAL, an initial screening of variables was performed before applying stepwise logistic regression. The variables were screened by univariable analysis, and only those with a $P$ value less than .05 were used as independent predictors in the logistic regression model (dependent variable: PAL).

Normally distributed variables were compared across groups using the Student $t$ test, and those without normal distribution were tested by the Mann-Whitney test. Categoric variables were tested by the chi-square test.

For the purpose of developing the aggregate score, a threshold effect was determined using receiver operating characteristic curves for those numeric variables to be included in the logistic regression. The logistic regression was validated by bootstrap analysis. Only predictors with a $P$ value less than .05 in more than $50 \%$ of 1000 bootstrap samples with the same number of observations as the original dataset were retained in the final model. ${ }^{13-15}$ Bootstrap is a technique of resampling with replacement through which new samples (1000 in our analysis) of patients are generated by randomly selecting individuals from the original database.

At each step of the simulation, every individual from the original database is again eligible to be selected, irrespective of whether he/she has already been sampled. Therefore, in each bootstrap sample, some of the original individuals may not be represented and others may be represented more than once. We have previously shown that the use of the entire dataset to develop the risk model and the application of bootstrap for its internal validation was superior to the traditional training and testing method of randomly splitting the database in a development and validation set. ${ }^{15}$ When applied to an external population, the model developed using the entire database and validated by bootstrap performed better than several models obtained by the training and testing methods. ${ }^{15}$ For this reason, we chose to use the former approach in this analysis.

The significant variables were used to construct an aggregate model according to the methodology described in previous studies. ${ }^{16,17}$ A score was assigned to each variable in the final model by proportionally weighting the regression coefficients and assigning 1 point to the smallest one (ie, if the smallest coefficient is 1.5 and another variable has a coefficient of 3,1 point is assigned to the first variable with smallest coefficient and 2 points to the other variable). A total score was then generated for each patient by summing the individual points assigned to each variable. The patients were finally grouped in risk classes according to their total scores and similar incidence of PAL within the group. The risk score was then applied to a sample of 2454 patients who underwent VATS lobectomy and were registered in the ESTS database from August 2015 to June 2016 with the aim to validate it in a population not used for its development. All tests were performed using Stata 12.0 statistical software (StataCorp LP, College Station, Tex).

\section{RESULTS}

The characteristics of the patients included in this study are shown in Table 1. PAL was observed in 504 patients $(9.9 \%)$. Patients with PAL also experienced a higher rate of cardiopulmonary complications compared with those without PAL $(12 \%$ vs $8.5 \%, P=.02)$. In patients with no other complications, the presence of PAL prolonged the postoperative hospital stay by 5 days compared with those without PAL (11.3 vs 6.2 days, $P<.0001$ ). The results of the univariable analysis to test the association of several variables with PAL are shown in Table 1. In particular, patients with PAL after VATS lobectomy had lower FEV1 $(P<.0001)$ and BMI $(P<.0001)$, and were more frequently male $(P<.0001)$.

For the purpose of this study, the numeric variables FEV1 and BMI were categorized by using receiver operating

TABLE 1. Characteristics of patients included in the analysis and comparison of baseline and surgical variables between those with and without prolonged air leak after video-assisted thoracoscopic surgery lobectomies

\begin{tabular}{lccccc}
\hline \multicolumn{1}{c}{ Variables } & $\begin{array}{c}\text { Entire cohort } \\
(\mathbf{n = 5 0 6 9 )}\end{array}$ & $\begin{array}{c}\text { With PAL } \\
\mathbf{( 5 0 4} \text { patients) }\end{array}$ & $\begin{array}{c}\text { Without PAL } \\
\mathbf{( 4 5 6 5} \text { patients) }\end{array}$ & OR (95\% CI) & $\begin{array}{c}\text { P value (between patients } \\
\text { with and without PAL) }\end{array}$ \\
\hline Age, y & $64(57-71)$ & $65.0(58-72)$ & $64.0(57-71)$ & & .2 \\
Sex male (n, \%) & $2862(56 \%)$ & $332(66 \%)$ & $2530(55 \%)$ & $1.55(1.3-1.9)$ & $<.0001$ \\
BMI $\left(\mathrm{kg} / \mathrm{m}^{2}\right)$ & $25.5(22.5-28)$ & $24.3(21.5-26.0)$ & $25.6(22.7-28.2)$ & & $<.0001$ \\
BMI $<18.5(\mathrm{n}, \%)$ & $180(3.6 \%)$ & $37(7.3 \%)$ & $143(3.1 \%)$ & $2.45(1.6-3.6)$ & $<.0001$ \\
FEV1\% & $84.4(72.9-96)$ & $81(66.8-93.1)$ & $84.4(73-96)$ & & $<.0001$ \\
FEV1 $<80 \%(\mathrm{n}, \%)$ & $1838(36 \%)$ & $234(46 \%)$ & $1604(35 \%)$ & $1.6(1.3-1.9)$ & .0001 \\
CAD $(\mathrm{n}, \%)$ & $417(8.2 \%)$ & $48(9.5 \%)$ & $369(8.1 \%)$ & $1.2(0.9-1.6)$ & .3 \\
ECOG score & $0(0-1)$ & $0(0-1)$ & $0(0-1)$ & & .4 \\
Diabetes $(\mathrm{n}, \%)$ & $188(3.7 \%)$ & $18(3.5 \%)$ & $170(3.7 \%)$ & $0.95(0.5-1.6)$ & .9 \\
Side right $(\mathrm{n}, \%)$ & $3035(60 \%)$ & $307(61 \%)$ & $2728(60 \%)$ & $1.04(0.9-1.3)$ & .6 \\
Site upper (n, \%) & $3215(63 \%)$ & $315(63 \%)$ & $2900(64 \%)$ & $0.96(0.8-1.2)$ & .7 \\
\hline
\end{tabular}

Results are expressed as medians and interquartile ranges for numeric variables and numbers and percentages of patients within the group for categoric variables. $P A L$, Prolonged air leak; $O R$, odds ratio; $C I$, confidence interval; $B M I$, body mass index; $F E V I$, forced expiratory volume in 1 second; $C A D$, coronary artery disease; $E C O G$, Eastern Cooperative Oncology Group score. 
TABLE 2. Results of the regression analysis (dependent variable: prolonged air leak)

\begin{tabular}{lccccc}
\hline Predictors & Coefficients & SE & $\boldsymbol{P}$ value & $\begin{array}{c}\text { Bootstrap } \\
\text { frequency }\end{array}$ & Score \\
\hline Intercept & -2.7 & 0.09 & & & \\
Male sex & 0.44 & 0.2 & $<.0001$ & $99.7 \%$ & 1 \\
FEV $1<80 \%$ & 0.4 & 0.1 & $<.0001$ & $99 \%$ & 1 \\
BMI $<18.5$ & 0.96 & 0.2 & $<.001$ & $99 \%$ & 2 \\
\hline
\end{tabular}

Bootstrap frequency: percentage of samples in which the variable resulted in $P<.05$. $S E$, Standard error; $F E V I$, forced expiratory volume in 1 second; $B M I$, body mass index.

characteristic analysis and selecting the best cutoff associated with PAL. The best cutoff values were $80 \%$ for FEV1 and $18.5 \mathrm{~kg} / \mathrm{m}^{2}$ for BMI, respectively. As a consequence, the variables used as independent predictors in the logistic regression analysis were FEV1 less than $80 \%$ predicted value, BMI less than $18.5 \mathrm{~kg} / \mathrm{m}^{2}$, and male sex.

A total of 1838 patients in this sample ( $36 \%$ of total) had an FEV1 lower than $80 \%$. Their PAL incidence was $12 \%$, and PAL occurrence in those with higher FEV1 was $7 \%$. PAL incidence in patients with FEV1 less than $70 \%$, $60 \%$, and $50 \%$ were $13 \%, 14 \%$, and $17 \%$, respectively.

A total of 180 patients $(3.6 \%$ of the total) had a BMI less than $18.5 \mathrm{~kg} / \mathrm{m}^{2}$. The PAL incidence in this group was $21 \%$. The PAL incidences in patients with BMI between 18.5 and $25 \mathrm{~kg} / \mathrm{m}^{2}$ or greater than $25 \mathrm{~kg} / \mathrm{m}^{2}$ were $11.3 \%$ and $8.3 \%$, respectively. All 3 variables were found to be associated with PAL after logistic regression analysis (Table 2).

The variables were proportionally scored according to their regression coefficients as follows: male sex, score $=1$; FEV1 less than $80 \%$ score $=1$; and BMI less than $18.5 \mathrm{~kg} / \mathrm{m}^{2}$ score $=2$ (Table 3). A PAL score was calculated for each patient by summing the individual scores assigned to each variable and ranged from 0 to 4 . According to their scores, patients were grouped into 4 classes with an incremental risk of PAL (chi-square test, $P<.0001$ ): class A (score 0 point, 1493 patients) $6.3 \%$, class B (score 1 point, 2240 patients) $9.9 \%$, class C (score 2 points, 1219 patients) $13 \%$, and class $\mathrm{D}$ (score $>2$ points,

TABLE 3. Breakdown of patient characteristics leading to class assignment

\begin{tabular}{lccrc}
\hline \multicolumn{1}{c}{ Variables } & $\begin{array}{c}\text { Class A } \\
\mathbf{n}=\mathbf{1 4 9 3}\end{array}$ & $\begin{array}{c}\text { Class B } \\
\mathbf{n}=\mathbf{2 2 4 0}\end{array}$ & $\begin{array}{c}\text { Class C } \\
\mathbf{n = 1 2 1 9}\end{array}$ & $\begin{array}{c}\text { Class D } \\
\mathbf{n = 1 1 7}\end{array}$ \\
\hline Male sex $(\mathrm{n}, \%)$ & 0 & $1647(74 \%)$ & $1156(95 \%)$ & $59(50 \%)$ \\
FEV1 $<80 \%(\mathrm{n}, \%)$ & 0 & $593(26 \%)$ & $1156(95 \%)$ & $89(76 \%)$ \\
BMI $<18.5 \mathrm{~kg} / \mathrm{m}^{2}$ & 0 & 0 & $63(5.2 \%)$ & $117(100 \%)$ \\
$(\mathrm{n}, \%)$ & & & & \\
\hline
\end{tabular}

Results are expressed as numbers and percentages of the total number of patients in that class of risk. FEVI, Forced expiratory volume in 1 second; BMI, body mass index.

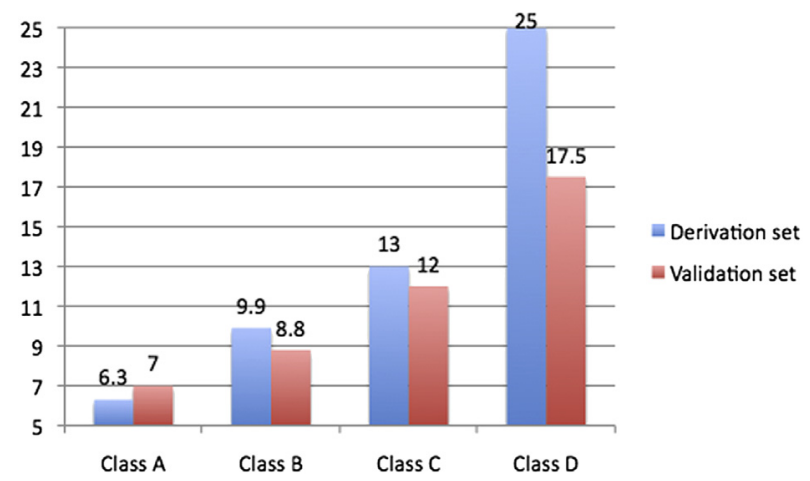

FIGURE 1. Incidence of PAL after VATS lobectomy according to the class of risk in the derivation and validation sets. Numbers in the $\mathrm{Y}$ axis indicate percentage of patients with PAL.

117 patients) $25 \%$ (Figure 1). Table 3 shows the distribution of patients with the 3 factors associated with PAL within each class of risk.

As expected, patients in the higher-risk classes had longer postoperative hospital stays (includes all patients in the analysis) (class A 6.4 days, class B 7.1 days, class C 8.4 days, class D 9.5 days; Kruskal-Wallis test, $P=.0001$ ). Dunn's pairwise comparison (with Bonferroni adjustment for multiple comparisons) of postoperative stay between risk groups showed that all differences between groups had $P$ values less than .0001 except for the difference between groups $\mathrm{C}$ and $\mathrm{D}$, which had a $P$ value of .02. Bootstrap analysis showed that patients in classes A (lowest risk class) and D (highest risk class) had a PAL incidence less than $7 \%$ in $86 \%$ of samples and greater than $20 \%$ in $87 \%$ of samples, respectively.

Finally, the score was applied to an independent sample of 2454 patients who underwent VATS lobectomy and were registered in the ESTS database after the completion of the analysis (from August 2015 to June 2016). In this group of patients not used to develop the model, the different classes of risk showed an incremental incidence of PAL $(P=.001)$ : class A (score 0 point, 440 patients) $7 \%$, class B (score 1 point, 1071 patients) $8.8 \%$, class C (score 2 points, 869 patients) $12.1 \%$, class D (score $>2$ points, 74 patients) $17.5 \%$ (Figure 1). When the logistic model was applied to the validation set, the classification accuracy for a predicted probability of PAL equal to or greater than $13 \%$ (corresponding to the incidence of PAL in class $\mathrm{C}$ of the derivation set) was $88 \%$.

\section{DISCUSSION}

\section{Background and Objective}

The application of VATS has been shown to reduce the incidence of morbidity rates particularly in high-risk patients, ${ }^{4-8,10}$ allowing for expansion of operability criteria in patients with early-stage lung cancer. ${ }^{18}$ The 
different case mix and some VATS-related technical modifications during the lobectomy procedure (ie, different approach to the fissures, more extensive use of blunt dissection of hilar structures, more liberal use of endostaplers) may have an impact on the occurrence of PAL. Thus, there is a need to develop a specific risk model for this VATS cohort of patients, with the aim of selecting those at higher risk for PAL as potential candidates for the application of intraoperative preventative measures. The aim of this study was to develop an aggregate risk score for predicting the occurrence of PAL after VATS lobectomy on the basis of the patients registered in the ESTS database.

\section{Database}

The choice to use a societal multi-institutional database has pros and cons. Certainly, when performing aggregate analyses on relatively rare events (eg, PAL after VATS lobectomy), the use of a population of thousands of patients allows the development of a more reliable predictive model. Moreover, the findings from analyses performed on large organizational databases (eg, ESTS database) are widely generalizable to the "real world" and represent contemporary information. ${ }^{19}$ On the other hand, the ESTS database was not designed for research purposes, but rather for quality improvement initiatives. As such, when performing specific analyses on selected outcomes, many critical variables potentially associated with the outcome of interest may be missing in the dataset. Moreover, as in all multi-institutional retrospective analyses, patient selection, surgical technique, and postoperative management may be variable in the different participating centers, introducing inherent biases into the analysis. However, the ESTS database includes most of the common risk factors associated with PAL in previously published single-center investigations and has a high data quality, as shown in previous specific investigations evaluating the quality of data in this specific database. ${ }^{20}$

\section{Main Findings}

In the present study, PAL was observed in 504 patients $(9.9 \%)$ of the total patients submitted to VATS lobectomies. This result is in line with previous articles focusing on the comparison of VATS and open approaches. ${ }^{10,11,21-23}$

PAL has been demonstrated to be one of the most important factors contributing to increased hospitalization length and overall hospital costs. ${ }^{24}$ In a recent article, Farjah and colleagues ${ }^{25}$ found that 90-day costs after VATS lobectomy are associated with prolonged hospital stay rather than health care use after discharge. One of the most important findings of the present analysis was that in patients with no other complications, the simple presence of PAL was associated with a postoperative hospital stay 5 days longer compared with those without PAL. In addition, patients in the highest class of risk remained in the hospital 3 days longer than those in the lowest class of risk. Therefore, the use of intraoperative measures to reduce the incidence of PAL or the volume of air leak (thus favoring earlier discharge with domiciliary chest drainage systems) in high-risk patients seems warranted even from a financial point of view.

The added cost of these measures, such as buttress material or surgical sealants, may become negligible in higher PAL risk groups because the cost of the measures may be increasingly offset by avoided hospital stay. Formalized economic analyses evaluating such measures in different PAL risk groups will be important to conduct in the future for hospital decision-makers.

The main finding of this study was the development of a simple PAL risk score based on 3 weighted factors: male gender, FEV1 less than $80 \%$, and BMI less than $18.5 \mathrm{~kg} / \mathrm{m}^{2}$. The score is seemingly effective at stratifying patients into 4 classes of risk. Patients with no risk factors had an incidence of PAL of $6 \%$, whereas patients in the highest classes of risk (classes $\mathrm{C}$ and $\mathrm{D}$ ) had 2-fold and 4-fold increases in PAL occurrence, respectively. This, as stated earlier, translated into a longer hospital stay for those in the highest classes of risk. Although a detailed financial analysis was not possible because of different economic systems present across countries contributing to the database, hospital stay is reputedly the main driver of hospital costs and can be taken as an indirect financial estimator.

As mentioned earlier, PAL scores have been published for open lobectomies. A few years ago, Brunelli and colleagues $^{3}$ developed a 4 -factor weighted aggregate score from a single-center database. Some of the variables were similar to those included in the present score. For instance, they found that low FEV1 and BMI were significantly associated with PAL. A reduced pulmonary function has been consistently reported as a risk factor for PAL. 3,26,27 The association between PAL and impaired pulmonary function can be explained by the increased susceptibility of a more fragile lung parenchyma to tear during lung dissection or manipulation and by a slower healing process. Likewise, a low BMI, a marker of malnutrition and reduced healing capacity, was found associated with increased risk of PAL by many studies. ${ }^{3,10,28,29}$ Nutritional assessment should be included in the routine preoperative selection to identify patients who can benefit from nutritional support programs.

Male gender and low BMI along with 7 other variables have been found to be associated with PAL in a previous risk model developed from the French Society of Cardiovascular and Thoracic Surgery database. ${ }^{2}$ These variables were recently validated in patients who underwent VATS lobectomy ${ }^{1}$; however, a specific score for VATS 
patients was not generated. The association between male gender and PAL is not entirely clear. In the present study, male gender likely represents a surrogate for other unaccounted for variables, such as smoking history or chronic obstructive pulmonary disease, which were not available in the database at the time of this analysis.

Another factor consistently associated with PAL in previous studies was the presence of pleural adhesions. ${ }^{10,30}$ Unfortunately, this variable is currently not recorded in the ESTS database and could not be factored into the present analysis. However, this study focuses on VATS lobectomies only, and extensive pleural adhesions may be a contraindication to VATS in some centers.

The other important use of this risk score may be as an informational tool during the preoperative informationprovision process. The risk score may inform preoperative patient counseling and allow clinicians to discuss with the patient the risk of developing a PAL and the likelihood of having a chest tube in place for a longer time or being discharged with a chest drain. Although we have shown that patient satisfaction is not directly related to postoperative complications, ${ }^{31}$ the importance of a tailored, informed, shared decision-making process may contribute to setting realistic expectations for patients undergoing VATS and may have further implication in their overall satisfaction with care.

Finally, a PAL risk score specific to VATS lobectomies would simplify the selection of patients for future efficacy studies on preventative interventions or chest tube management, making the interpretation of results across different investigations more consistent and reproducible, especially in the presence of paucity of randomized trials in this area.

\section{Clinical Implications and Future Perspectives}

The main clinical implication of this study is the possibility of applying this score to select patients receiving VATS lobectomy who are at high risk of developing PAL. This may serve a clinical and investigational purpose: With a VATS approach, the submersion test to verify air leak at the end of the procedure is sometimes unreliable, and the use of this risk score can help in selecting patients at increased risk of PAL who may benefit most of the application of sealants or other intraoperative preventative measures. A financial analysis should be performed in each local health care system to estimate whether the cost of applying preventative measures in all high-risk patients would be offset by the reduction of PAL incidence and hospital stay costs in the $15 \%$ to $25 \%$ of patients in whom PAL is expected to occur. The score can be used to select high-risk patients to be included in future randomized trials investigating the cost-effectiveness of sealants or other measures aimed at reducing the incidence of air leak and improving the reliability and meaningfulness of these analyses.

\section{Study Limitations and Strengths}

As discussed, all the results generated from multiinstitutional databases should be interpreted taking into consideration the restraints and structural limitations of these datasets (eg, the lack of specific variables of interest, variability in patient selection, surgical technique, and postoperative management across participating centers).

For the purpose of this study, we did not have access to information regarding specific VATS techniques or modalities (eg, the rate of conversion, the approach to the fissures). Furthermore, in the ESTS database, there is no information regarding the number and type of endostaplers used or the use of energy devices to develop the fissures. Another important aspect that is missing from the ESTS database is the use of intraoperative measures to minimize air leakage, such as pleural tenting, applying buttressed staple devices, or using sealants. The ESTS database does not contain any information regarding the type of chest drainage systems used (digital vs traditional) or the type of postoperative chest tube management (suction vs no suction). The future implementation of a specific section for minimally invasive procedures will likely obviate these problems. Another limitation of the database is the absence of the duration of air leak expressed as numeric variable, which would have allowed testing different thresholds of PAL. A low BMI was present in only $3.5 \%$ of patients. This may represent a critical factor because this predictor may be under-represented in certain population strata. In the current dataset, the value of 18.5 represented the best cutoff; however, future analyses are warranted aimed at redefining this threshold to increase its representativeness. Finally, the ESTS database is a voluntary database. Although more than 200 European Hospitals contribute data into the registry, they represent less than $30 \%$ of all centers practicing thoracic surgery in Europe. This may introduce some problems of representativeness and generalizability of the data used for the analysis.

The study is based on a large multicenter organizational database, which ensures that the findings are contemporary and reflect the real clinical world. In addition, we performed an external validation using a sample of patients not used to develop the original model. This validation confirmed the generalizability of the risk score. The model developed and validated in this study includes only 3 readily available variables making it user-friendly and easy to implement in the clinical practice.

\section{CONCLUSIONS}

We created an aggregate risk score to stratify the incidence of PAL after VATS lobectomy. The score can be used for patient counseling and to identify those patients who can benefit from additional intraoperative preventative measures. 


\section{Webcast}

You can watch a Webcast of this AATS meeting presentation by going to: http://webcast.aats.org/2016/Video/ Tuesday/05-17-16_Ballroom_IV_1610_Pompili-800.mp4.

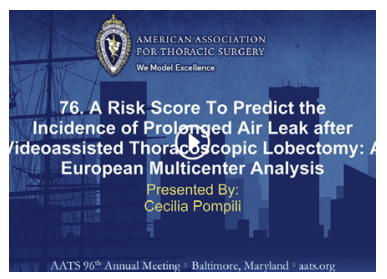

\section{Conflict of Interest Statement}

Authors have nothing to disclose with regard to commercial support.

The authors thank all of the centers contributing to the ESTS Database for their continuous commitment and active participation (http://www.ests.org/collaboration/database_contributors_list.aspx). Their contribution is pivotal to the advancement of science in our specialty and to ensure high-quality care to our patients. The authors thank Dr Danilo Pellicano for invaluable help in managing the data.

\section{References}

1. Orsini B, Baste JM, Gossot D, Berthet JP, Assouad J, Dahan M, et al. Index of prolonged air leak score validation in case of video-assisted thoracoscopic surgery anatomical lung resection: results of a nationwide study based on the French national thoracic database, EPITHOR. Eur J Cardiothorac Surg. 2015;48: 608-11.

2. Rivera C, Bernard A, Falcoz PE, Thomas P, Schmidt A, Bénard S, et al. Characterization and prediction of prolonged air leak after pulmonary resection: a nationwide study setting up the index of prolonged air leak. Ann Thorac Surg. 2011;92:1062-8.

3. Brunelli A, Varela G, Refai M, Jimenez MF, Pompili C, Sabbatini A, et al. A scoring system to predict the risk of prolonged air leak after lobectomy. Ann Thorac Surg. 2010;90:204-9.

4. Begum SS, Papagiannopoulos K, Falcoz PE, Decaluwe H, Salati M, Brunelli A. Outcome after video-assisted thoracoscopic surgery and open pulmonary lobectomy in patients with low VO2 max: a case-matched analysis from the ESTS database. Eur J Cardiothorac Surg. 2016;49:1054-8.

5. Zhang R, Ferguson MK. Video-assisted versus open lobectomy in patients with compromised lung function: a literature review and meta-analysis. PLoS One. 2015; 10:e0124512.

6. Fernando HC, Landreneau RJ, Mandrekar SJ, Nichols FC, DiPetrillo TA, Meyers BF, et al. Alliance for Clinical Trials in Oncology. Analysis of longitudinal quality-of-life data in high-risk operable patients with lung cancer: results from the ACOSOG Z4032 (Alliance) multicenter randomized trial. J Thorac Cardiovasc Surg. 2015;149:718-26.

7. Burt BM, Kosinski AS, Shrager JB, Onaitis MW, Weigel T. Thoracoscopic lobectomy is associated with acceptable morbidity and mortality in patients with predicted postoperative forced expiratory volume in 1 second or diffusing capacity for carbon monoxide less than $40 \%$ of normal. J Thorac Cardiovasc Surg. 2014; 148:19-28; discussion 28-9.e1.

8. Ceppa DP, Kosinski AS, Berry MF, Tong BC, Harpole DH, Mitchell JD, et al. Thoracoscopic lobectomy has increasing benefit in patients with poor pulmonary function: a Society of Thoracic Surgeons Database analysis. Ann Surg. 2012;256: 487-93.

9. Shaw JP, Dembitzer FR, Wisnivesky JP, Litle VR, Weiser TS, Yun J, et al. Videoassisted thoracoscopic lobectomy: state of the art and future directions. Ann Thorac Surg. 2008;85:S705-9.

10. Falcoz PE, Puyraveau M, Thomas PA, Decaluwe H, Hürtgen M, Petersen RH, et al. ESTS Database Committee and ESTS Minimally Invasive Interest Group.
Video-assisted thoracoscopic surgery versus open lobectomy for primary nonsmall-cell lung cancer: a propensity-matched analysis of outcome from the European Society of Thoracic Surgeon database. Eur J Cardiothorac Surg. 2016; 49:602-9.

11. Paul S, Altorki NK, Sheng S, Lee PC, Harpole DH, Onaitis MW, et al. Thoracoscopic lobectomy is associated with lower morbidity than open lobectomy: a propensity-matched analysis from the STS database. J Thorac Cardiovasc Surg. 2010;139:366-78.

12. Fernandez FG, Falcoz PE, Kozower BD, Salati M, Wright CD, Brunelli A. The Society of Thoracic Surgeons and the European Society of Thoracic Surgeons general thoracic surgery databases: joint standardization of variable definitions and terminology. Ann Thorac Surg. 2015;99:368-76.

13. Blackstone EH. Breaking down barriers: helpful breakthrough statistical methods you need to understand better. J Thorac Cardiovasc Surg. 2001;122: 430-9.

14. Grunkemeier GL, Wu YX. Bootstrap resampling method: something for nothing? Ann Thorac Surg. 2004;1142-4.

15. Brunelli A, Rocco G. Internal validation of risk models in lung resection surgery: bootstrap versus training and test sampling. J Thorac Cardiovasc Surg. 2006;131 1243-7.

16. Passman RS, Gingold DS, Amar D, Lloyd-Jones D, Bennett CL, Zhang H, et al. Prediction rule for atrial fibrillation after major noncardiac thoracic surgery. Ann Thorac Surg. 2005;79:1698-703.

17. Hristova R, Pompili C, Begum S, Salati M, Kefaloyannis M, Tentzeris V, et al. An aggregate score to predict the risk of large pleural effusion after pulmonary lobectomy. Eur J Cardiothorac Surg. 2015;48:72-6.

18. Howington JA, Blum MG, Chang AC, Balekian AA, Murthy SC Treatment of stage I and II non-small cell lung cancer: diagnosis and management of lung cancer, 3rd ed: American College of Chest Physicians Evidence-Based Clinical Practice Guidelines. Chest. 2013;143(5 Suppl): e278S-313S.

19. Murthy SC, Blackstone EH. Research based on big data: the good, the bad, and the ugly. J Thorac Cardiovasc Surg. 2016;151:629-30.

20. Salati M, Falcoz PE, Decaluwe H, Rocco G, Van Raemdonck D, Varela G, et al ESTS Database Committee. The European Thoracic Data Quality Project: an aggregate data quality score to measure the quality of international multiinstitutional databases. Eur J Cardiothorac Surg. 2016;49:1470-5.

21. Flores RM, Park BJ, Dycoco J, Aronova A, Hirth Y, Rizk NP, et al. Lobectomy by video-assisted thoracic surgery (VATS) versus thoracotomy for lung cancer. $J$ Thorac Cardiovasc Surg. 2009;138:11-8.

22. Whitson BA, Groth SS, Duval SJ, Swanson SJ, Maddaus MA. Surgery for early stage non-small cell lung cancer: a systematic review of the video-assisted thoracoscopic surgery versus thoracotomy approaches to lobectomy. Ann Thorac Surg. 2008;86:2008-18.

23. Nwogu CE, D'Cunha J, Pang H, Gu L, Wang X, Richards WG, et al. Alliance for Clinical Trials in Oncology. VATS lobectomy has better perioperative outcomes than open lobectomy: CALGB 31001, an ancillary analysis of CALGB 140202 (Alliance). Ann Thorac Surg. 2015;99:399-405.

24. Varela G, Jiménez MF, Novoa N, Aranda JL. Estimating hospital costs attributable to prolonged air leak in pulmonary lobectomy. Eur J Cardiothorac Surg. 2005;27:329-33.

25. Farjah F, Backhus LM, Varghese TK, Mulligan MS, Cheng AM, AlfonsoCristancho R, et al. Ninety-day costs of video-assisted thoracic surgery versus open lobectomy for lung cancer. Ann Thorac Surg. 2014;98:191-6.

26. Abolhoda A, Liu D, Brooks A, Burt M. Prolonged air leak following radical upper lobectomy: an analysis of incidence and possible risk factors. Chest. 1998; 113:1507-10.

27. Brunelli A, Monteverde M, Borri A, Salati M, Marasco RD, Fianchini A. Predictors of prolonged air leak after pulmonary lobectomy. Ann Thorac Surg. 2004;77: 1205-10.

28. Thomas PA, Berbis J, Falcoz PE, Le Pimpec-Barthes F, Bernard A, Jougon J, et al; EPITHOR Group. National perioperative outcomes of pulmonary lobectomy for cancer: the influence of nutritional status. Eur J Cardiothorac Surg. 2014;45:652-9.

29. Fiorelli A, Vicidomini G, Mazzella A, Messina G, Milione R, Di Crescenzo VG, et al. The influence of body mass index and weight loss on outcome of elderly patients undergoing lung cancer resection. Thorac Cardiovasc Surg. 2014;62: 578-87.

30. Bernard A, Rivera C, Falcoz PE, Vicaut E, Thomas P, Dahan M. Application of model score of prolonged air leak in the French database. Ann Thorac Surg. 2011; 92:1548-50. 
31. Pompili C, Tiberi M, Salati M, Refai M, Xiumé F, Brunelli A. Patient satisfaction with health-care professionals and structure is not affected by longer hospital stay and complications after lung resection: a case-matched analysis. Interact Cardiovasc Thorac Surg. 2015;20:236-41.

Key Words: prolonged air leak, video-assisted thoracoscopic surgery, lobectomy, risk modeling, risk score, VATS lobectomy

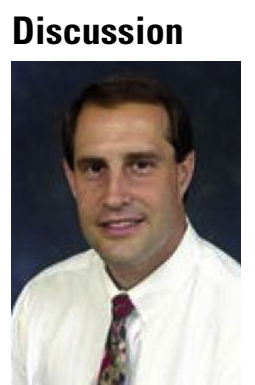

Dr R. Cerfolio (Birmingham, Ala). This article is well written, and the presentation was outstanding, but you have given me an index of which I can't change what the patient comes with. Your second to the last bullet is the key. What do I do with this information? Let's talk about how this is going to clinically affect our practice. Am I really going to use buttressing devices that cost $\$ 400$ and still leak because the air leak is from another part in the lung? Am I going to use glue that costs $\$ 800$ ? Am I going to put in 2 chest tubes? How is it going to make me get better care for the patient?

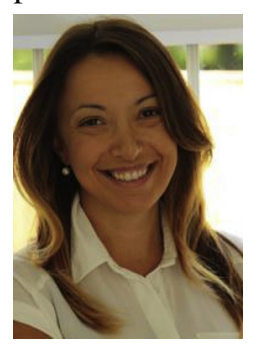

Dr Pompili (Leeds, UK). The main clinical implication of this study is to select high-risk patients. We all know that the intraoperative preventative measures have a cost, and they are not always available in all the countries, and I'm specifically speaking about the European countries where there are not many chances for all patients to have these measures or glue.

Dr Cerfolio. Well, we can't afford it in the United States either. Will your next study look at one of these variables? Is that what this will launch to? What do you plan to do with the data?

Dr Pompili. I think we can plan to select the high-risk patients and then apply some management protocol in terms of intraoperative measures, such as preventative or postoperative measures, because these apply to postoperative measures in terms of chest tube management. So high-risk patients will be selected by this score.

Dr Cerfolio. We are all putting them on water seal pretty much. How are you going to manage their chest tubes differently?

Dr Pompili. The chest tube is not a problem because we are using 1 chest tube in any case. I think we can focus more on the intraoperative and postoperative in terms of chest tube management.
Dr Cerfolio. We have seen a lot of reports at this meeting that the databases have been questionable, and this is a problem I have with large databases, and I have watched our specialty go from really good data in uni-institutions to a whole bunch of bad data because the $\mathrm{N}$ is 15,000 , and I really have a problem with articles that come out of that. What are the limitations with this? I never saw carbon monoxide lung diffusion capacity (DLCO) percent. Did I miss that when I read your article? Did you really not have the DLCO percent in these patients?

Dr Pompili. No. First, I would like to stress the importance of reading the Murthy and Blackstone article ${ }^{19}$ about the good, bad, and ugly of organizational databases. It is one of the best articles about this. That is something related to the limitations of the organizational database. We had DLCO for only $30 \%$ of cases, so we needed to exclude it. All the variables included in these analyses had at least 90\% presence of completeness.

Dr Cerfolio. Was there any other variable that you wanted to have besides DLCO percent?

Dr Pompili. The pleural adhesions will be something to look at because they have been demonstrated to be associated with PAL. We can assume in this type of specific analysis that VATS lobectomy is a contraindication in general, but it will be of interest to study the pleural adhesions in this VATS group.

Dr Cerfolio. But then you are going to have to score it and give a scoring and a grading and have to do it scientifically. The last question is the use of digital air leak systems. I know that your group has written about it. We did 12 or 15 years ago. They have some cost, but they have some benefit in getting tubes out of people quicker when you send them home with them. What is your experience with digital air leaks?

Dr Pompili. In this specific study, we did not have any information about digital drains. Obviously, the selection of high-risk patients can be something to look at. Even for the postoperative management with digital drains, we do not have the availability for all of our patients, so we can select patients who can benefit more from digital drains with this score.

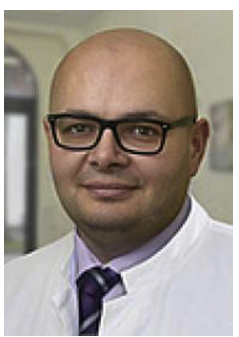
Dr S. Bolukbas (Wuppertal, Germany). At least in Germany, almost all patients have an FEV1 less than $80 \%$ undergoing lobectomy for lung cancer. Therefore, do you have a receiver operating characteristic analysis for a specific value where you find good actors and bad actors?

Dr Pompili. Unfortunately, that was the same answer for the BMI. We selected these 2 numeric variables with the receiver operating characteristic analysis, and that was the best cutoff for the FEV1 and the BMI associated with PAL. That is the reason why we chose the less than $80 \%$. 


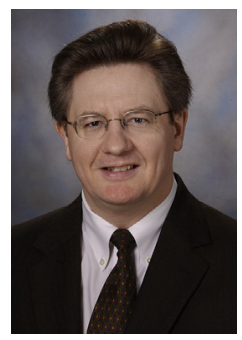

Dr G. Walsh (Houston, Tex). Just a thought to get your idea about a potential future study. Perhaps in your identified higher-risk patients you could randomize to a pleural tent or not. It doesn't cost any more except for a few extra minutes of operating room time to do the pleural tent.
Dr Pompili. I agree. The pleural tent can be one of the added features that we can use in a specific future randomized trial to identify the high-risk patient with this score. So high-risk patients identified can be randomized between pleural tent or not or other preventative measures.

Readers who found these articles interesting may also like to read the following papers found in recent and future issues of our sister publications, Seminars in Thoracic and Cardiovascular Surgery and Operative Techniques in Thoracic and Cardiovascular Surgery!

\section{Thoracic: Oncology}

Computer-Aided Nodule Assessment and Risk Yield Risk Management of Adenocarcinoma: The Future of Imaging? Finbar Foley. Semin Thoracic Surg 2016:120-6.

Robotic Esophagectomy for Cancer: Early Results and Lessons Learned Robert J. Cerfolio. Semin Thoracic Surg 2016:160-9. Improving Quality: When Surgeons Take the Bit Between the Teeth, Patients Win! Katie S. Nason. Semin Thoracic Surg 2016:170-1. Second Primary Lung Cancers Demonstrate Better Survival with Surgery than Radiation Emanuela Taioli. Semin Thoracic Surg 2016:195-200.

Surgery is the Optimum Local Therapeutic Modality for Second Primary Lung Cancer Jeffrey L. Port. Semin Thoracic Surg 2016:201-2.

The Next Generation of Mesothelioma Surgeons Roundtable Discussion Harvey Pass. Semin Thoracic Surg 2016:203-20.

Novel Methods of Intraoperative Localization and Margin Assessment of Pulmonary Nodules Jane Keating. Semin Thoracic Surg 2016:127-36.

Is Close Surveillance Indicated for Indolent Cancers? The Carcinoid Story Sudish C. Murthy. Semin Thoracic Surg 2016: 541-6.

Prognostic Relevance of Lymph Node Regression After Neoadjuvant Chemoradiation for Esophageal Cancer Annouck Philippron. Semin Thoracic Surg 2016:549-58.

Esophageal Cancer: Improvements in Treatment, Staging, and Now Prognostic Indicators? (Invited Editorial Commentary) Mark F. Berry. Semin Thoracic Surg 2016:559-60.

Immune Response after Radiofrequency Ablation and Surgical Resection in Non-Small Cell Lung Cancer Thomas Schneider. 585-92.

Outcome of Patients With pN2 "Potentially Resectable“ NSCLC Who Underwent Surgery After Induction Chemotherapy Lorenzo Spaggiari. Semin Thoracic Surg 2016:593-602.

Complete Postobstructive Lung Atelectasis Due to Malignant Central Airway Obstruction Does not Exclude Curative Chance Grigoris Stratakos. Semin Thoracic Surg 2016:603-5.

Evolution in the Surgical Care of Patients With Non-Small Cell Lung Cancer in the Mid-South Quality of Surgical Resection Cohort Nicholas R. Faris. Semin Thoracic Surg 2016: In press.

Paradigm for Detecting Silent Thoracic Aneurysm Disease John A. Elefteriades. Semin Thoracic Surg 2016: In press. 